\title{
Mars moon ephemerides after 14 years of Mars Express data
}

\author{
V. Lainey ${ }^{1}$, A. Pasewaldt ${ }^{2}$, V. Robert ${ }^{3,1}$, P. Rosenblatt ${ }^{4}$, R. Jaumann ${ }^{2}$, J. Oberst $^{2}$, T. Roatsch ${ }^{2}$, K. Willner ${ }^{2}$, \\ R. Ziese ${ }^{5}$, and W. Thuillot ${ }^{1}$
}

\author{
${ }^{1}$ IMCCE, Observatoire de Paris, PSL Research University, CNRS, Sorbonne Universités, UPMC Univ. Paris 06, Univ. Lille, France \\ e-mail: lainey@imcce.fr \\ 2 German Aerospace Center (DLR), Rutherfordstr. 2, 12489 Berlin-Adlershof, Germany \\ ${ }^{3}$ IPSA, 63 bis boulevard de Brandebourg, 94200 Ivry-sur-Seine, France \\ ${ }^{4}$ Laboratoire De Planétologie Et Géodynamique, Bâtiment 4, 2 Chemin de la Houssinière, 44300 Nantes, France \\ 5 Technical University of Berlin, Straße des 17. Juni 135, 10623 Berlin, Germany
}

Received 11 September 2020 / Accepted 26 December 2020

\begin{abstract}
The Mars Express (MEX) mission has been successfully operated around Mars since 2004. Among many results, MEX has provided some of the most accurate astrometric data of the two Mars moons, Phobos and Deimos. We present new ephemerides of the Mars moons benefitting from all previously published astrometric data to the most recent MEX SRC data. Observations from 1877 until 2018 and including spacecraft measurements from Mariner 9 to MEX were included. Assuming a homogeneous interior, we fitted the forced libration amplitude of Phobos simultaneously with the Martian tidal $k_{2} / Q$ ratio and the initial state of the moons. Our solution of the physical libration $1.09 \pm 0.01$ degrees deviates notably from the homogeneous solution. Considering the very low error bar, however, this may essentially suggest the necessity to consider higher order harmonics with an improved rotation model in the future. While most data could be successfully fitted, we found a disagreement between the Mars Reconnaissance Orbiter and the Mars Express astrometric data at the kilometer level, probably associated with a biased phase correction. The current solution precision is expected at the level of a few hundred meters for Phobos and several hundred meters for Deimos for the coming years. The real accuracy of our new ephemerides will have to be confirmed by comparison with independent observational means.
\end{abstract}

Key words. ephemerides - astrometry - celestial mechanics

\section{Introduction}

Space astrometry of the natural satellites allows us to reach a precision that is far better than that of telescopic observations. For Phobos, we can access a short history of these telescopic observations in Pascu et al. (2014). In order to exploit the combination of space- and ground-based data to compute ephemerides, we received funding from the European FP7 program and coordinated the European Satellite Partnership for Computing Ephemerides (ESPaCE; Thuillot et al. 2012) consortium from 2011 to 2015. ESPaCE brought together seven laboratories (IMCCE-Paris Observatory; the Royal Observatory of Belgium, ROB; the Joint Institute for VLBI in Europe, JIVE-ERIC; the Technical University of Berlin, TUB; the German Aerospace Center, DLR; the French Space Center, CNES; and the Technical University of Delft, TUD) that focused on different activities related to the positioning of spacecraft and natural satellites such as digitizing of old telescopic observations, the exploitation of spacecraft radio-tracking data to reconstruct spacecraft orbits, and the development of planetary moons ephemerides. The ESPaCE portal ${ }^{1}$ provides access to further information. In this context, a fruitful collaboration has been established between the ESPaCE consortium and the MaRS radio-science and the HRSC camera teams of the MEX mission, in preparation of the close approach of Phobos in December 2013.

On 29 December 2013, Mars Express (MEX) approached the Mars moon Phobos up to $45 \mathrm{~km}$, which was closer than

\footnotetext{
http://espace.oma.be
}

ever before. To guarantee the highest precision on the position of Phobos, an astrometric observation campaign of Phobos was performed with the MEX onboard camera Super-Resolution Channel (SRC). Several dozen observations were performed and reduced using the USNO CCD Astrograph Catalog (UCAC4) to provide strong constraints on the Phobos orbit in the International Celestial Reference Frame (ICRF; Pasewaldt et al., in prep.). New ephemerides of the Martian satellites were developed as part of the consortium. They are presented here.

In Sect. 2 we detail the numerical model we used. In Sect. 3 we present the set of observations we used. The next section shows the astrometric results and provides physical parameters of interest, such as the amplitude of the forced libration of Phobos and the tidal $k_{2} / Q$ ratio of Mars. In Sect. 5 we discuss an issue we encountered when we considered Mars Reconnaissance Orbiter (MRO) observations. The last section is devoted to the precision of the extrapolation and to a comparison with former ephemerides.

\section{Modeling}

We used a very similar approach as described in Lainey et al. (2007). In particular, we used the NOE numerical code to model the orbits of Phobos and Deimos. The Mars rotation model we considered was the one of Konopliv et al. (2016) with the Mars gravity field MRO120D that we truncated at degree and order 12. Table 1 provides the numerical values of these coefficients. The masses of Mars (42828.3750104 $\mathrm{km}^{3} \mathrm{sec}^{-2}$ ), Phobos 
Table 1. Mars gravity field we used (MRO120D; Konopliv et al. 2016) truncated at degree and order 12.

\begin{tabular}{|c|c|}
\hline \multicolumn{2}{|c|}{ Harmonic coefficients } \\
\hline \multirow[t]{3}{*}{$C_{2,0}, C_{3,0}, \ldots C_{12,0}$} & $-0.87502209245370 \mathrm{E}-03-0.11897015037300 \mathrm{E}-040.51290958301340 \mathrm{E}-05-0.17267702404260 \mathrm{E}-05$ \\
\hline & 0.13463714866160E-05 0.10598968092880E-05 0.14437523554120E-06 -0.28755906339710E-06 \\
\hline & $0.72663209592840 \mathrm{E}-06-0.26620541678020 \mathrm{E}-060.26016282936860 \mathrm{E}-06$ \\
\hline \multirow[t]{3}{*}{$C_{21}, C_{31}, \ldots, C_{12,1}$} & 0.40223333063820E-09 0.38049981991010E-05 0.42163911582170E-05 0.48384215630660E-06 \\
\hline & 0.18023583692130E-05 0.13749994266810E-05 -0.13253837279380E-06 0.42096311969260E-06 \\
\hline & $0.92399054314190 \mathrm{E}-06-0.81684431270170 \mathrm{E}-06-0.11268252543490 \mathrm{E}-05$ \\
\hline \multirow[t]{3}{*}{$C_{22}, C_{32}, \ldots, C_{12,2}$} & $-0.84633026559830 \mathrm{E}-04-0.15947431923720 \mathrm{E}-04-0.95306695299840 \mathrm{E}-06-0.42981760456790 \mathrm{E}-05$ \\
\hline & $0.86171342848250 \mathrm{E}-06 \quad 0.28139783170420 \mathrm{E}-050.18104244435100 \mathrm{E}-050.11387318459420 \mathrm{E}-05$ \\
\hline & $0.69959052072370 \mathrm{E}-08-0.31359320991050 \mathrm{E}-06-0.97535431722040 \mathrm{E}-07$ \\
\hline \multirow[t]{3}{*}{$C_{33}, C_{43}, \ldots, C_{12,3}$} & 0.35056298360330E-04 0.64568519841300E-05 0.33126670085550E-05 0.95567075435960E-06 \\
\hline & $0.88048981361710 \mathrm{E}-06-0.12070336086800 \mathrm{E}-05-0.99365116894820 \mathrm{E}-06-0.29903288633400 \mathrm{E}-06$ \\
\hline & $-0.13030274194510 \mathrm{E}-05-0.14420200318390 \mathrm{E}-05$ \\
\hline \multirow[t]{3}{*}{$C_{44}, C_{54}, . . C_{12,4}$} & $0.30824936247700 \mathrm{E}-06-0.46407608474120 \mathrm{E}-05$ 0.10087553624920E-05 0.24689899049820E-05 \\
\hline & $0.15882837683240 \mathrm{E}-050.29497879998410 \mathrm{E}-06-0.12139330875140 \mathrm{E}-05-0.15741226303010 \mathrm{E}-05$ \\
\hline & $\begin{array}{ll}-0.10141072962050 \mathrm{E}-06\end{array}$ \\
\hline \multirow[t]{2}{*}{$C_{55}, \ldots C_{12,5}$} & $-0.44492645268970 \mathrm{E}-050.16578866158220 \mathrm{E}-05-0.19168169008940 \mathrm{E}-06-0.27917524692450 \mathrm{E}-05$ \\
\hline & $-0.22719046508670 \mathrm{E}-050.42141118528680 \mathrm{E}-060.13564965343170 \mathrm{E}-050.72031532166240 \mathrm{E}-06$ \\
\hline \multirow[t]{2}{*}{$C_{66}, \ldots C_{12,6}$} & $0.27622296666680 \mathrm{E}-05-0.55960104736480 \mathrm{E}-06-0.91440928257930 \mathrm{E}-060.80860626285270 \mathrm{E}-06$ \\
\hline & $0.66960055718320 \mathrm{E}-06-0.24475256306890 \mathrm{E}-06-0.40343909794620 \mathrm{E}-06$ \\
\hline \multirow[t]{2}{*}{$C_{77}, \ldots C_{12,7}$} & $0.44039558349960 \mathrm{E}-06-0.47364667411900 \mathrm{E}-06-0.61795403730490 \mathrm{E}-060.31785454600440 \mathrm{E}-06$ \\
\hline & 0.66045729925750E-06 0.40444336054540E-06 \\
\hline \multirow[t]{2}{*}{$C_{88} \ldots C_{12,8}$} & $-0.31065725923520 \mathrm{E}-06$ 0.12080798860860E-05 0.54059795600680E-06 -0.11820682984680E-05 \\
\hline & $\begin{array}{ll}20.16039741594790 E-05\end{array}$ \\
\hline$C_{9,9}, \ldots C_{12,9}$ & $-0.11719047495280 \mathrm{E}-05-0.14575391837510 \mathrm{E}-05-0.41520987784620 \mathrm{E}-060.70845500937310 \mathrm{E}-06$ \\
\hline$C_{10,10 \ldots C_{12,10}}$ & $-0.27477718238890 \mathrm{E}-060.33329692241000 \mathrm{E}-060.48850428613000 \mathrm{E}-06$ \\
\hline$C_{11,11}, C_{12,11}$ & $-0.44950881784560 \mathrm{E}-070.86475982548170 \mathrm{E}-06$ \\
\hline$C_{12,12}$ & $-0.66853201533900 \mathrm{E}-08$ \\
\hline \multirow{3}{*}{$S_{21}, S_{31}, \ldots, S_{12,1}$} & $0.23031838535520 \mathrm{E}-100.25177117707630 \mathrm{E}-040.37632643561220 \mathrm{E}-050.21231129753950 \mathrm{E}-05$ \\
\hline & $-0.15185193991260 \mathrm{E}-05-0.22737194866050 \mathrm{E}-060.75052081765850 \mathrm{E}-06-0.49021207335710 \mathrm{E}-06$ \\
\hline & $0.22320069479760 \mathrm{E}-06-0.22130696733310 \mathrm{E}-06-0.51245929517270 \mathrm{E}-07$ \\
\hline \multirow[t]{3}{*}{$S_{22}, S_{32}, \ldots, S_{12,2}$} & $0.48939418321670 \mathrm{E}-040.83623939784670 \mathrm{E}-05-0.89807968418080 \mathrm{E}-05-0.11656954440860 \mathrm{E}-05$ \\
\hline & 0.14691007371520 E-05 -0.62967694381360E-06 0.50705823024380E-06 0.38219982478050E-06 \\
\hline & $-0.11157916028810 \mathrm{E}-05-0.99401812075920 \mathrm{E}-060.53304503764570 \mathrm{E}-06$ \\
\hline \multirow[t]{3}{*}{$S_{33}, S_{43}, \ldots, S_{12,3}$} & $0.25571325457370 \mathrm{E}-04-0.19377212284160 \mathrm{E}-060.27144097785790 \mathrm{E}-060.33292558689320 \mathrm{E}-06$ \\
\hline & $-0.39698286606070 \mathrm{E}-06-0.13413009168640 \mathrm{E}-05-0.10076169517160 \mathrm{E}-050.44355180981810 \mathrm{E}-06$ \\
\hline & $0.76528953740340 \mathrm{E}-060.31477016571890 \mathrm{E}-06$ \\
\hline \multirow[t]{3}{*}{$S_{44}, S_{54}, . . S_{12,4}$} & $-0.12873056977380 \mathrm{E}-04-0.33815536222490 \mathrm{E}-050.26386569471530 \mathrm{E}-05-0.42245529297430 \mathrm{E}-06$ \\
\hline & 0.14812074290120E-06 0.16235822767760E-05 -0.69716893597150E-07 -0.64448534121760E-06 \\
\hline & $\begin{array}{lll}0.12358719897030 \mathrm{E}-06 & 0.06\end{array}$ \\
\hline \multirow{2}{*}{$S_{55}, \ldots S_{12,5}$} & $0.37804789409520 \mathrm{E}-05$ 0.16226764849180E-05 -0.13585219042100E-05 -0.16297926112810E-05 \\
\hline & $-0.15493068736430 \mathrm{E}-05-0.10629285253530 \mathrm{E}-05$ 0.89908740763190E-06 0.10016903702080E-05 \\
\hline \multirow[t]{2}{*}{$S_{66}, \ldots S_{12,6}$} & $0.82135333243850 \mathrm{E}-06-0.19013643905730 \mathrm{E}-05-0.17899324707530 \mathrm{E}-050.57773114546310 \mathrm{E}-06$ \\
\hline & $0.11171248335330 \mathrm{E}-050.20644450708130 \mathrm{E}-07-0.16298585248040 \mathrm{E}-05$ \\
\hline \multirow[t]{2}{*}{$S_{77}, \ldots S_{12,7}$} & $-0.17756701426830 \mathrm{E}-05$ 0.16446964596680E-05 0.86891992653820E-06 -0.62023200497360E-06 \\
\hline & -0.86497561721440E-06 -0.11283348722240E-06 \\
\hline \multirow[t]{2}{*}{$S_{88} \ldots S_{12,8}$} & $-0.25028184874420 \mathrm{E}-06-0.14644731038890 \mathrm{E}-060.82057425718930 \mathrm{E}-060.76703348424800 \mathrm{E}-06$ \\
\hline & 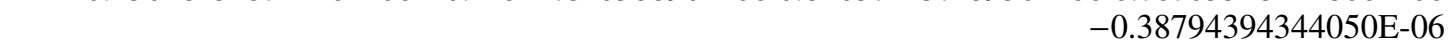 \\
\hline$S_{9,9}, \ldots S_{12,9}$ & $-0.65779217295200 \mathrm{E}-06-0.14635579222260 \mathrm{E}-05-0.41944370089590 \mathrm{E}-060.47674076749600 \mathrm{E}-06$ \\
\hline$S_{10,10 \ldots S_{12,10}}$ & 0.75328042634720E-06 0.19666657150790E-05 0.13782862550090E-05 \\
\hline$S_{11,11}, S_{12,11}$ & $-0.32345607088980 \mathrm{E}-06-0.16550385123550 \mathrm{E}-05$ \\
\hline$S_{12,12}$ & $-0.89210620377340 \mathrm{E}-07$ \\
\hline
\end{tabular}

Notes. Coefficients are normalized, and the Mars radius is equal to $3396.0 \mathrm{~km}$.

$\left(7.11 \times 10^{-4} \mathrm{~km}^{3} \mathrm{sec}^{-2}\right)$, Deimos $\left(9.46 \times 10^{-5} \mathrm{~km}^{3} \mathrm{sec}^{-2}\right)$, and the Mars Love number $k_{2}(0.169)$ were adopted from this gravity solution. The planetary ephemerides were INPOP19a (Fienga et al. 2020), which introduced the perturbations of the Sun, the Moon, and all the other planets.
Three amendments on the dynamics were introduced in comparison to Lainey et al. (2007). The first two were the introduction of general relativity and tidal cross effects (Lainey et al. 2017) that consist in the cross action of the tidal bulges raised on Mars by different tide-raising bodies. While rather 
negligible at the current level of accuracy for Mars, we added these effects for completeness. Tides on the Mars moons were neglected.

A third but important dynamical effect was the introduction of the forced libration on the rotation of Phobos (Jacobson 2010). This perturbation introduces a secular effect on the periapsis of Phobos (Borderies \& Yoder 1990; Lainey et al. 2019) that can barely be masked in the fitting procedure. The secular drift, associated with the quadrupole field of Phobos on its periapsis (or any moon in a somewhat similar configuration) was reported by Borderies \& Yoder (1990) and recalled by Jacobson (2010) to be

$$
\begin{aligned}
\Delta \varpi= & \frac{3}{2}\left(\frac{R}{a}\right)^{2}\left[J_{2}-2 c_{22}\left(5-\frac{4 A}{e}\right)\right] n t \\
& +\frac{3}{2}\left(\frac{R}{a}\right)^{2} \frac{\left(J_{2}+6 c_{22}\right)}{e} \sin (\mathcal{M}),
\end{aligned}
$$

where $a, e, n, \mathcal{M}$, and $\varpi$ are the traditional osculating Keplerian semimajor axis, eccentricity, mean motion, and mean anomaly, and $R, J_{2}=-c_{20}$ and $c_{22}$ denote the mean radius and unnormalized gravity coefficients of Phobos. Here we explicitly see the action of the libration amplitude $A$ on the periapsis. Our $A$ is $-A$ in Jacobson (2010). This action of the quadrupole field of the satellite is just one effect on the pericenter. The precession of the pericenter is mainly due to the flattening of Mars.

Because the gravity field of Phobos is hard to determine directly from the current data (Yang et al. 2019), we relied on Willner et al. (2014), who used the shape of Phobos and homogeneous density hypothesis to derive the gravity field of Phobos. They obtained $c_{20}=-0.066127$ and $c_{22}=0.009917$ for the first gravity field coefficients, assuming a radius of $14.0 \mathrm{~km}$ for Phobos.

\section{Observation sets}

Most data sets we used were preivously processed in Lainey et al. (2007) and Jacobson (2010), and benefited greatly from the astrometric catalog of Morley (1989). A large astrometric database is available on the Natural Satellites DataBase (NSDB) server (Arlot \& Emelyanov 2009). Some of the old measurements were extremely biased and therefore were eventually not included in the fit. Moreover, ground observations with residuals larger than 2 arcseconds were removed. A rejection criterion beyond $3 \sigma$ was applied to ground observations. Last, spacecraft data were weighted in the same way as in Lainey et al. (2019). Here we present two important new data sets that were not available to the former works.

\subsection{Observations from digitized plates}

Following the discovery of Phobos and Deimos and the detection of the long-suspected secular accelerations in their longitudes (Sharpless 1945), the United States Naval Observatory (USNO) began a 30-yr (1967-1997) program of photographic observations of the Martian satellites (Pascu 1977, 1978, 1979, 2012). These observations were among the most accurate (Morley 1989) and were used to support all space reconnaissance projects of the Martian system.

Photographic observations were begun in 1967 and continued at every opposition through 1997 . They were taken with the USNO 61-inch astrometric reflector in Flagstaff, Arizona, and the USNO 26-inch refractor in Washington, D.C. with the use of special filters. Several Schott 5 inch $\times 7$ inch $\times 3$ mm GG14 (yellow) filters were polished optically flat. In the center of each, a small, thin metallic nichrome film with an optical density of about 3.0 was deposited by evaporation. The GG14 was chosen to accommodate both telescopes, and nichrome was chosen because it transmits neutrally in the visual bandwidth. The function of the small nichrome filter was to reduce the intensity of the planetary image to that of the satellites, producing a measurable image of the planetary disk. A number of Kodak emulsions were used, including 103aJ, 103aG, and IIIaJ. More details about the observations and astrometric results are available in Robert et al. (2015).

Four hundred twenty-five plates were selected and transmitted to the Royal Observatory of Belgium (ROB) to be digitized (Robert et al. 2011; de Cuyper et al. 2011). Each plate contains two to three exposures shifted in the RA direction. Measured $(x, y)$ plate positions were corrected for instrumental and spherical effects, and the reductions were performed using four or six suitable constant functional models (Robert et al. 2011, 2014) to provide equatorial (RA, Dec) astrometric positions of the planet and its satellites. The digitizations and measurements resulted in 777 positions of Mars, 640 positions of Phobos, and 704 positions of Deimos.

The observed positions of Mars, Phobos, and Deimos were compared with their theoretically computed positions given by the DE430 planetary ephemeris (Folkner et al. 2014) and NOE MarsSatV1_0 satellite ephemerides (Lainey et al. 2007). The key point was that the NOE MarsSatV1_0/DE430 astrometric residuals $(\mathrm{O}-\mathrm{C})$ for all observations had an rms of 47.8 mas, 60.5 mas and 50.7 mas for Mars, Phobos, and Deimos, respectively. The overall intersatellite RMS (O-C) is 39 mas. These rms correspond to the observation accuracies over $30 \mathrm{yr}$, providing observations that are similar in accuracy to old space data, but have a more Gaussian profile and a longer time span.

\subsection{New MEX/SRC data}

MEX is in a highly elliptical and nearly polar orbit of Mars that reaches well beyond the almost circular path of Phobos (Jaumann et al. 2007). Because of the asphericity of the planetary gravitational field, its longitude of the ascending node is drifting westward and its argument of pericenter is drifting in the direction opposite to the spacecraft motion. At the same time, its orbital period is similar to that of the inner satellite of Mars, resulting in several flyby opportunities in consecutive orbits at intervals of about five to six months. Whenever a close encounter between the space probe and this Martian moon occurs, highresolution observations of Phobos by the onboard cameras are planned, see Sect. 3.2.1. In the past years, new opportunities of observing Phobos and Deimos in conjunction with other Solar System objects or stars (so-called mutual events, see Sect. 3.2.2) were identified and planned more regularly. These observations do not necessarily require the proximity to the body (Ziese and Willner 2018).

The Super Resolution Channel (SRC) is part of the High Resolution Stereo Camera (HRSC) onboard MEX. It features a compact Maksutov-Cassegrain optical system, a $1 \mathrm{~K}$ by $1 \mathrm{~K}$ interline-transfer $\mathrm{CCD}$, and fast read-out electronics (Oberst et al. 2008).

\subsubsection{Close encounters}

During an approach maneuver, the camera is pointed to a fixed location on the celestial sphere and is rotated around the boresight axis such that the lower and upper image borders are 
parallel to the relative velocity vector of Phobos with respect to the spacecraft. In the usual eight-image sequences, the first and the last pictures are long-time exposures to detect the faint light of background stars. Exposure times of the images in between are adjusted to the brighter Phobos surface. Pointing corrections in sample and line are derived from star images and then interpolated linearly for the pictures of Phobos (Willner et al. 2008).

For the purpose of astrometry, SRC images have been focussed using an image-derived point spread function in a Richardson-Lucy deconvolution (Michael \& Neukum 2009). The image positions of stars were determined by fitting a 2D Gaussian profile to the pixel value distributions (Duxbury 2012, priv. comm.). Pointing corrections were derived with respect to the predictions of reference star positions based on the UCAC4 catalog. The directions to Phobos were measured by applying the limb-fit approach based on the latest 3D shape model by Willner et al. (2014).

Between May 2013 and March 2014 a larger number of flybys than usual were performed in order to support the Phobos gravity field experiment. To do this, data from 38 approach maneuvers with distances ranging from 350 to $14000 \mathrm{~km}$ were evaluated. To summarize, 340 astrometric observations of Phobos in right ascension and declination coordinates were provided. The estimated uncertainties vary from hundreds of meters up to one kilometer (Pasewaldt et al., in prep.).

The uncertainty estimation is still incomplete so far because of occasional spacecraft jitter during image acquisition (see Pasewaldt et al. 2015 for details). For sequences with continuous observations of reference stars or other celestial objects, this causes no problem. For several eight-image series with only two star pictures, however, an additional uncertainty is introduced that is hard to quantify. Therefore we separated our Phobos measurements into those performed during linear variation and those carried out during nonlinear variation in camera pointing.

In addition to the measurement of reference objects, the CCD line coordinate of a Phobos position could provide information on the presence or absence of spacecraft oscillations during image recording. Based on the imaging geometry described above, this should be almost constant throughout a sequence. From at least three successive Phobos observations we can calculate two line coordinate differences.

While only positive or negative differences were classified as linear variations, alternating positive and negative differences were categorized as nonlinear variations in pointing. If in addition the prefit residuals deviated by more than $3 \sigma$ from the arithmetic mean, we marked this as an outlier. For series with only two Phobos measurements, we were unable to determine whether the outlier was related to a nonlinear change in pointing. Furthermore, in some images we fit the shape model-derived limb to only very short limb-point arcs. This classification is not an equivalent substitute for a proper weight estimate, but it gives at least a suggestion for the following evaluation of our data.

\subsubsection{Mutual event observations}

Longer imaging sequences of mutual events showing either both Martian moons or one of the moons with Jupiter or Saturn in the background were analyzed by Ziese and Willner (2018). These observations can be obtained more frequently than direct observations during flybys. Moreover, a wide range of MEX orbit positions are possible. However, in contrast to close encounter images, Phobos and Deimos can also cross the image plane diagonally. The moons are only partially visible at times as well.

To determine the locations of the bodies within the image, simulated images were computed. The simulations were based on the ephemerides model mar097, a rotational model by Stark et al. (2017) (see Archinal 2018; Archinal et al. 2019), and on shape models of Phobos and Deimos derived by Willner et al. (2014) and Thomas et al. (2000), respectively. To achieve a better agreement with the observation, the simulation was convolved with a point spread function describing the image distortion of the SRC. Here a subset of the point spread function derived by Duxbury et al. (2011) was applied. Matching the illuminated simulation with the observation provided the body lines and sample coordinates with subpixel accuracy. For images showing either Phobos and/or Deimos with a far distant object, the derived right ascension and declination of the moon (as seen from MEX) were determined. The exhaustive list of MEX data we used is given in Table 2. In particular, because of a current limitation of our software, mutual events evolving both moons simultaneously (i.e., without Jupiter or Saturn) were not included in the current solution.

\section{Adjustment results}

All data were fit using the RMS of each data set as a priori uncertainty. Data points higher than 2 arcsec for ground-based observations were systematically removed. Then, a $3 \sigma$ rejection criterion was applied. For spacecraft observations, we used the published estimated precision. However, to assess a better weight, we rescaled these uncertainties by a scalar chosen to obtain about $66 \%$ of astrometric residuals within the $1 \sigma$ level for each satellite and coordinate.

We provide in Figs. 1-3 and Tables 3-6 the astrometric residuals of all ground- and space-based data we used to develop the ephemerides and initial conditions of Phobos and Deimos after the fit. The high precision of the reduction of photographic plates and the new MEX data is confirmed, with typical residuals at a level of 40-50 mas and 300 meters. The combination of the existence of two Mars moons with the use of intersatellite fitting often provides both opposite mean values and similar $\sigma$. This feature is absent when the two moons could always not be observed simultaneously, as indicated by a different number of observation per moon. We note that the MEX residuals may be associated with an error on the astrometric calibration of the field and the error on the spacecraft position. In particular, it was found that the long imaging sequence of mutual events (see Sect. 3.2) introduced a significant bias in the fitting procedure. While the jitter effect of the spacecraft could be removed thanks to the mutual event opportunity, the error on the position of MEX in space remained. An error of about a few hundred meters on the reconstructed orbit of MEX was found, which is much higher than the initially estimated error, which was at a level of 2025 meters (Rosenblatt et al. 2008). The increase in the error on the MEX position in 3D space apparently arose from an unfortunate consequence of the new tracking strategy of MEX. Since 2011, ESA has decreased the amount of tracking data around periapsis, entailing a poorer constraint on the motion of MEX.

Based on the new sets, we formally gained an order of magnitude on the uncertainty $(1 \sigma)$ of the Martian tidal quality factor and the physical libration of Phobos. Assuming $k_{2}=0.169$, we obtained $Q=93.68 \pm 0.18$. Because the eccentricity of the Phobos orbit is low (lower than $2 \%$ ), the secular variation in its 
Table 2. Mean $(v)$ and standard deviation $(\sigma)$ on right ascension and declination for each satellite.

\begin{tabular}{|c|c|c|c|c|c|c|}
\hline Observations & $\begin{array}{l}v_{\alpha \cos \delta} \\
(\mathrm{km})\end{array}$ & $\begin{array}{l}\sigma_{\alpha \cos \delta} \\
(\mathrm{km})\end{array}$ & $\begin{array}{r}v_{\delta} \\
(\mathrm{km})\end{array}$ & $\begin{array}{r}\sigma_{\delta} \\
(\mathrm{km})\end{array}$ & $N$ & Satellite \\
\hline Mariner 9 (Duxbury \& Callahan 1989) & $\begin{array}{l}-7.2594 \\
-0.7282\end{array}$ & $\begin{array}{l}7.3718 \\
4.1653\end{array}$ & $\begin{array}{l}-6.2897 \\
-3.4240\end{array}$ & $\begin{array}{r}8.1181 \\
4.3704\end{array}$ & $\begin{array}{c}48,48 \\
14,14\end{array}$ & $\begin{array}{l}\text { Phobos } \\
\text { Deimos } \\
\end{array}$ \\
\hline Viking 1 (Duxbury \& Callahan 1988) & $\begin{array}{r}-0.4629 \\
0.7174\end{array}$ & $\begin{array}{l}9.8178 \\
3.4617\end{array}$ & $\begin{array}{l}-0.1971 \\
-1.9736\end{array}$ & $\begin{array}{l}8.7993 \\
4.9500\end{array}$ & $\begin{array}{r}132,132 \\
19,19\end{array}$ & $\begin{array}{l}\text { Phobos } \\
\text { Deimos }\end{array}$ \\
\hline Viking 2 (Duxbury \& Callahan 1988) & $\begin{array}{r}2.6933 \\
-1.4065\end{array}$ & $\begin{array}{l}7.7604 \\
3.3602\end{array}$ & $\begin{array}{r}-5.1783 \\
-0.7202\end{array}$ & $\begin{array}{r}8.0789 \\
11.9342\end{array}$ & $\begin{array}{l}32,32 \\
80,80\end{array}$ & $\begin{array}{l}\text { Phobos } \\
\text { Deimos }\end{array}$ \\
\hline Phobos 2 (Kolyuka et al. 1991) & $\begin{array}{r}-0.3396 \\
3.9608\end{array}$ & $\begin{array}{r}0.8558 \\
15.3037\end{array}$ & $\begin{array}{r}-0.2277 \\
12.0239\end{array}$ & $\begin{array}{r}0.5194 \\
11.2175\end{array}$ & $\begin{array}{r}37,37 \\
8,8\end{array}$ & $\begin{array}{l}\text { Phobos } \\
\text { Deimos }\end{array}$ \\
\hline MEX ((Willner et al. 2008); priv. comm.) & $\begin{array}{r}-0.0867 \\
0.0000\end{array}$ & $\begin{array}{l}0.4485 \\
0.0000\end{array}$ & $\begin{array}{l}0.0548 \\
0.0000\end{array}$ & $\begin{array}{l}0.4973 \\
0.0000\end{array}$ & $\begin{array}{r}135,135 \\
0,0\end{array}$ & $\begin{array}{l}\text { Phobos } \\
\text { Deimos }\end{array}$ \\
\hline MEX (Pasewaldt et al. 2012) & $\begin{array}{l}0.0000 \\
0.0395\end{array}$ & $\begin{array}{l}0.0000 \\
1.1084\end{array}$ & $\begin{array}{r}0.0000 \\
-0.3801\end{array}$ & $\begin{array}{l}0.0000 \\
1.0322\end{array}$ & $\begin{array}{r}0,0 \\
136,136\end{array}$ & $\begin{array}{l}\text { Phobos } \\
\text { Deimos }\end{array}$ \\
\hline MEX (CP) (Pasewaldt et al. 2015) & $\begin{array}{r}-0.3113 \\
0.0000\end{array}$ & $\begin{array}{l}0.5400 \\
0.0000\end{array}$ & $\begin{array}{l}0.0383 \\
0.0000\end{array}$ & $\begin{array}{l}0.9030 \\
0.0000\end{array}$ & $\begin{array}{r}130,130 \\
0,0\end{array}$ & $\begin{array}{l}\text { Phobos } \\
\text { Deimos }\end{array}$ \\
\hline MEX (LF) (Pasewaldt et al. 2015) & $\begin{array}{r}-0.1153 \\
0.0000\end{array}$ & $\begin{array}{l}0.4126 \\
0.0000\end{array}$ & $\begin{array}{l}0.2043 \\
0.0000\end{array}$ & $\begin{array}{l}0.9995 \\
0.0000\end{array}$ & $\begin{array}{r}27,27 \\
0,0\end{array}$ & $\begin{array}{l}\text { Phobos } \\
\text { Deimos }\end{array}$ \\
\hline MEX-flyby-linear-outlier (Pasewaldt et al., in prep.) & $\begin{array}{r}-0.3070 \\
0.0000\end{array}$ & $\begin{array}{l}0.0529 \\
0.0000\end{array}$ & $\begin{array}{l}0.4736 \\
0.0000\end{array}$ & $\begin{array}{c}0.0411 \\
0.0000\end{array}$ & $\begin{array}{l}3,3 \\
0,0\end{array}$ & $\begin{array}{l}\text { Phobos } \\
\text { Deimos }\end{array}$ \\
\hline MEX-flyby-linear (Pasewaldt et al., in prep.) & $\begin{array}{r}-0.0872 \\
0.0000\end{array}$ & $\begin{array}{l}0.2683 \\
0.0000\end{array}$ & $\begin{array}{l}0.0488 \\
0.0000\end{array}$ & $\begin{array}{l}0.3004 \\
0.0000\end{array}$ & $\begin{array}{r}64,64 \\
0,0\end{array}$ & $\begin{array}{l}\text { Phobos } \\
\text { Deimos }\end{array}$ \\
\hline MEX-flyby-nonlinear (Pasewaldt et al., in prep.) & $\begin{array}{r}0.2143 \\
0.0000\end{array}$ & $\begin{array}{l}0.4333 \\
0.0000\end{array}$ & $\begin{array}{l}0.1653 \\
0.0000\end{array}$ & $\begin{array}{l}0.3507 \\
0.0000\end{array}$ & $\begin{array}{r}22,22 \\
0,0\end{array}$ & $\begin{array}{l}\text { Phobos } \\
\text { Deimos }\end{array}$ \\
\hline MEX-flyby-nonlinear-outlier (Pasewaldt et al., in prep.) & $\begin{array}{r}-0.0224 \\
0.0000\end{array}$ & $\begin{array}{l}0.2974 \\
0.0000\end{array}$ & $\begin{array}{l}0.2931 \\
0.0000\end{array}$ & $\begin{array}{l}0.1644 \\
0.0000\end{array}$ & $\begin{array}{l}8,8 \\
0,0\end{array}$ & $\begin{array}{l}\text { Phobos } \\
\text { Deimos }\end{array}$ \\
\hline MEX-flyby-shortlimb (Pasewaldt et al., in prep.) & $\begin{array}{r}-0.4813 \\
0.0000 \\
\end{array}$ & $\begin{array}{l}0.2241 \\
0.0000\end{array}$ & $\begin{array}{l}0.3064 \\
0.0000 \\
\end{array}$ & $\begin{array}{l}0.4526 \\
0.0000\end{array}$ & $\begin{array}{l}6,6 \\
0,0\end{array}$ & $\begin{array}{l}\text { Phobos } \\
\text { Deimos } \\
\end{array}$ \\
\hline MEX-flyby-outlier (Pasewaldt et al., in prep.) & $\begin{array}{r}-0.8951 \\
0.0000\end{array}$ & $\begin{array}{l}0.0746 \\
0.0000\end{array}$ & $\begin{array}{r}-0.5160 \\
0.0000\end{array}$ & $\begin{array}{l}0.1332 \\
0.0000\end{array}$ & $\begin{array}{l}3,3 \\
0,0\end{array}$ & $\begin{array}{l}\text { Phobos } \\
\text { Deimos }\end{array}$ \\
\hline MEX-Dei-Saturn-Ziese & $\begin{array}{r}0.0000 \\
-0.2029\end{array}$ & $\begin{array}{r}0.0000 \\
0.1710\end{array}$ & $\begin{array}{r}0.0000 \\
-0.2820\end{array}$ & $\begin{array}{l}0.0000 \\
0.0931\end{array}$ & $\begin{array}{r}0,0 \\
64,64\end{array}$ & $\begin{array}{l}\text { Phobos } \\
\text { Deimos }\end{array}$ \\
\hline MEX-Pho-Saturn-Ziese & $\begin{array}{l}0.1904 \\
0.0000\end{array}$ & $\begin{array}{l}0.2440 \\
0.0000\end{array}$ & $\begin{array}{r}0.1121 \\
0.0000\end{array}$ & $\begin{array}{l}0.1287 \\
0.0000\end{array}$ & $\begin{array}{r}295,295 \\
0,0\end{array}$ & $\begin{array}{l}\text { Phobos } \\
\text { Deimos }\end{array}$ \\
\hline MEX-Dei-Jup-Ziese & $\begin{array}{r}0.0000 \\
-1.0983\end{array}$ & $\begin{array}{l}0.0000 \\
0.0782\end{array}$ & $\begin{array}{l}0.0000 \\
0.8743\end{array}$ & $\begin{array}{l}0.0000 \\
0.0184\end{array}$ & $\begin{array}{r}0,0 \\
14,14\end{array}$ & $\begin{array}{l}\text { Phobos } \\
\text { Deimos }\end{array}$ \\
\hline MEX-Pho-Jup-Ziese & $\begin{array}{l}0.2920 \\
0.0000\end{array}$ & $\begin{array}{l}0.0269 \\
0.0000\end{array}$ & $\begin{array}{r}-0.0773 \\
0.0000\end{array}$ & $\begin{array}{r}0.0319 \\
0.0000\end{array}$ & $\begin{array}{r}50,50 \\
0,0\end{array}$ & $\begin{array}{l}\text { Phobos } \\
\text { Deimos }\end{array}$ \\
\hline
\end{tabular}

Notes. Both angles are multiplied by the distance spacecraft-moon to obtain kilometers. $N$ is the number of observations by a satellite. Pasewaldt et al. (2015) determined the positions of Phobos using control point (CP) and/or limb point (LF) measurements. The former are based on the satellite control network, a set of identifiable surface features that are evenly distributed over the body's surface and define its reference system. Recent MEX SRC measurements are separated into observations made during linear and nonlinear pointing variations. If the observation prefit residuals deviated by more than $3 \sigma$ from the mean value, they were additionally categorized as an outlier. For only a few outliers we were unable to clarify whether they were related to nonlinear variations in pointing. Some measurements are based on fits of the shape-model-derived limb to only very short limb-point arcs in the image (see also Sect. 3.2.1).

semimajor axis can be easily approximated using (Kaula 1964)

$\frac{\mathrm{d} a}{\mathrm{~d} t} \simeq-\frac{3 k_{2} m n R^{5}}{Q M a^{4}}$,

where $m$ and $M$ denote the mass of Phobos and Mars, respectively. From Eq. (2) we obtain for the secular acceleration on longitude

$\frac{1}{2} \frac{\mathrm{d} n}{\mathrm{~d} t} \simeq+\frac{9 n^{2} k_{2} m R^{5}}{4 Q M a^{5}}$.

Using the approximation $n^{2} a^{3} \simeq G M$ and the value $9378 \mathrm{~km}$ for the Phobos semimajor axis, we obtain for the tidal acceleration 

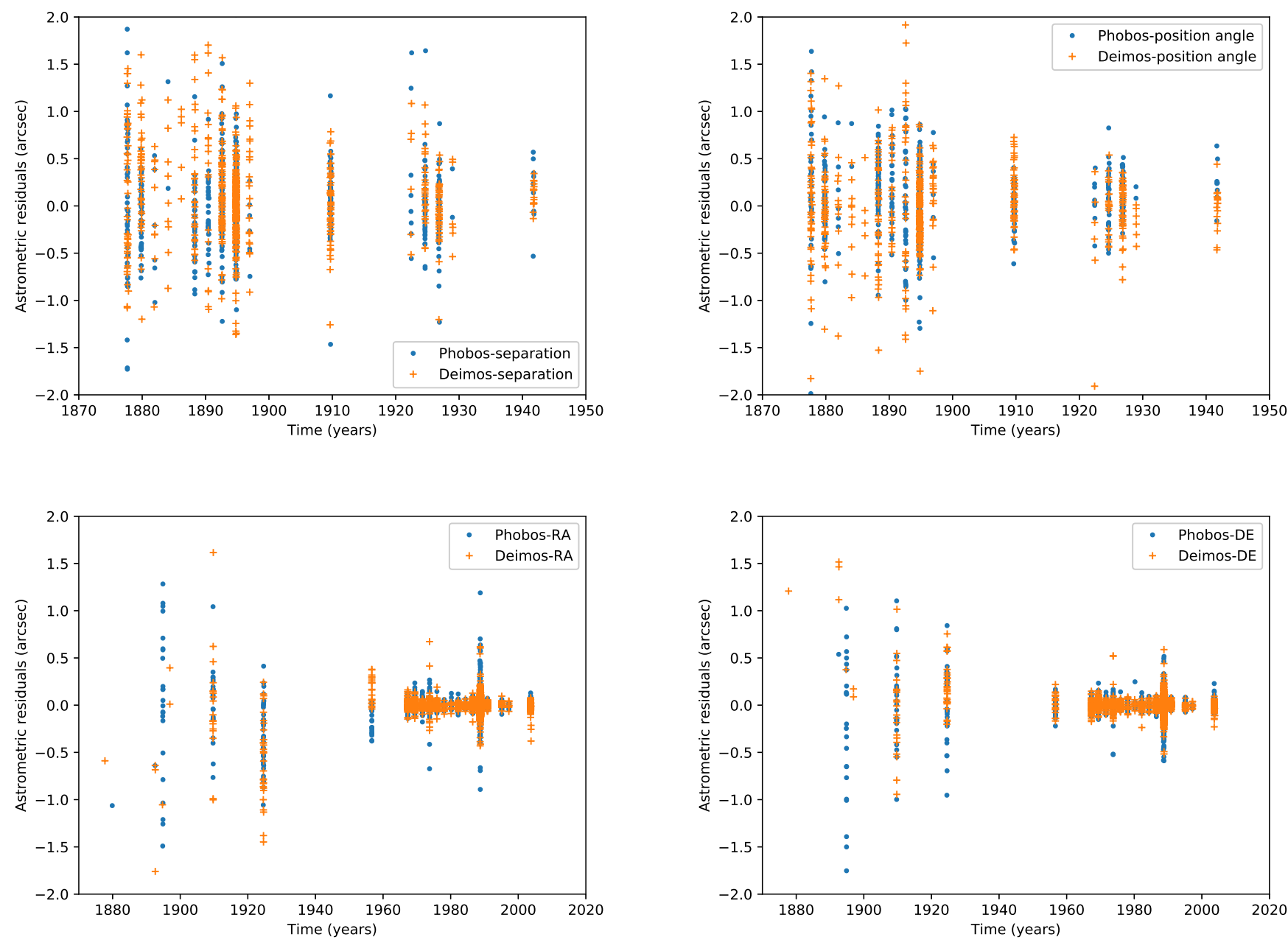

Fig. 1. Astrometric residuals after the fit between the model and the ground-based observations for Phobos and Deimos (top: separation and position angle data, and bottom: right ascension and declination data). The initial positions and velocities of the satellites, the Martian dissipation quality factor $Q$, and the forced libration amplitude of Phobos were fit. The position angle was multiplied by the separation to provide residuals in arcsec.
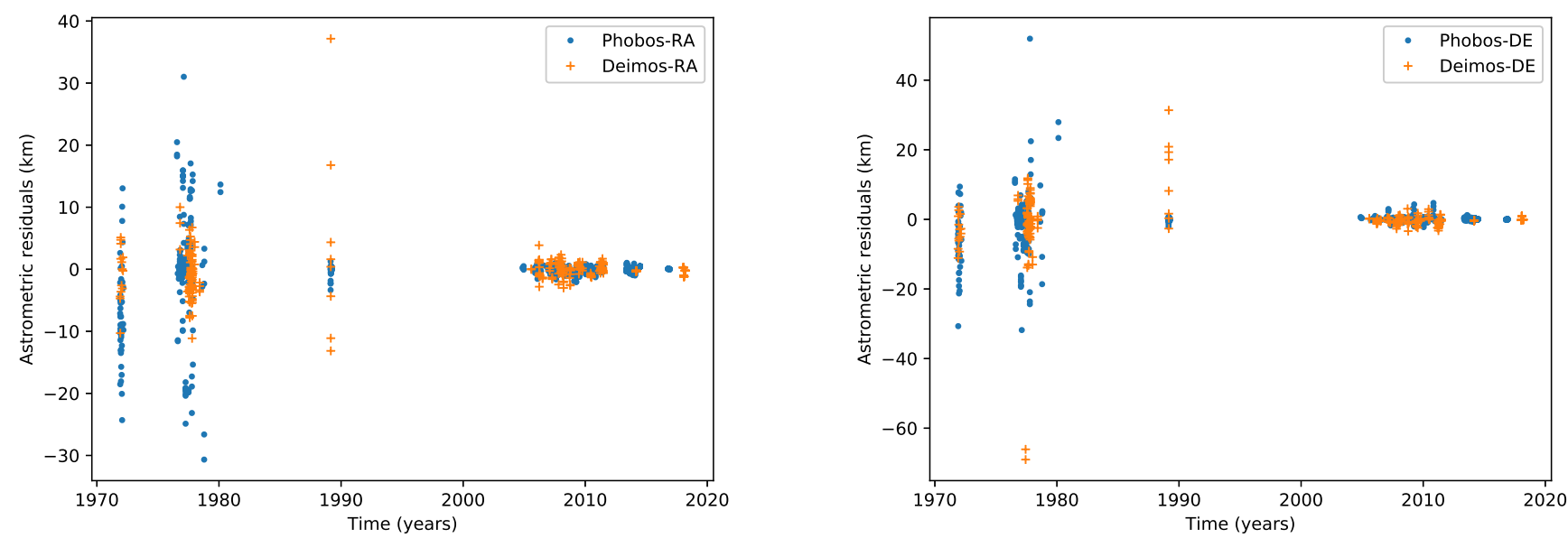

Fig. 2. Differences in distance after the fit between the model and the spacecraft observations for Phobos and Deimos (left: right ascension; right: declination). The initial positions and velocities of the satellites (at epoch J2000, i.e., Julian day 2451545.0 ), the Martian dissipation quality factor $Q$, and the forced libration amplitude of Phobos were fit.

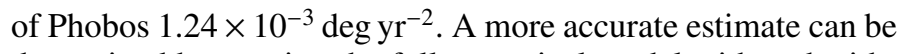
determined by running the full numerical model with and without tidal dissipation inside Mars. This provides the tidal accel-

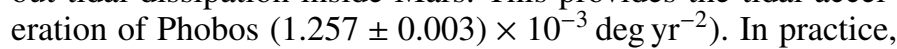
this orbital decay and associated longitude acceleration allows us to estimate $Q$ for Mars. We recall that we did not include higher Love numbers $\left(k_{3}, k_{4}\right.$, etc.) in our fit. Our estimate of $Q$ related to $k_{2}$ should therefore be more considered as a lumped $Q$ parameter. Considering the high precision we now obtain on this tidal parameter (lower than $0.2 \%$ ), it would probably make sense 

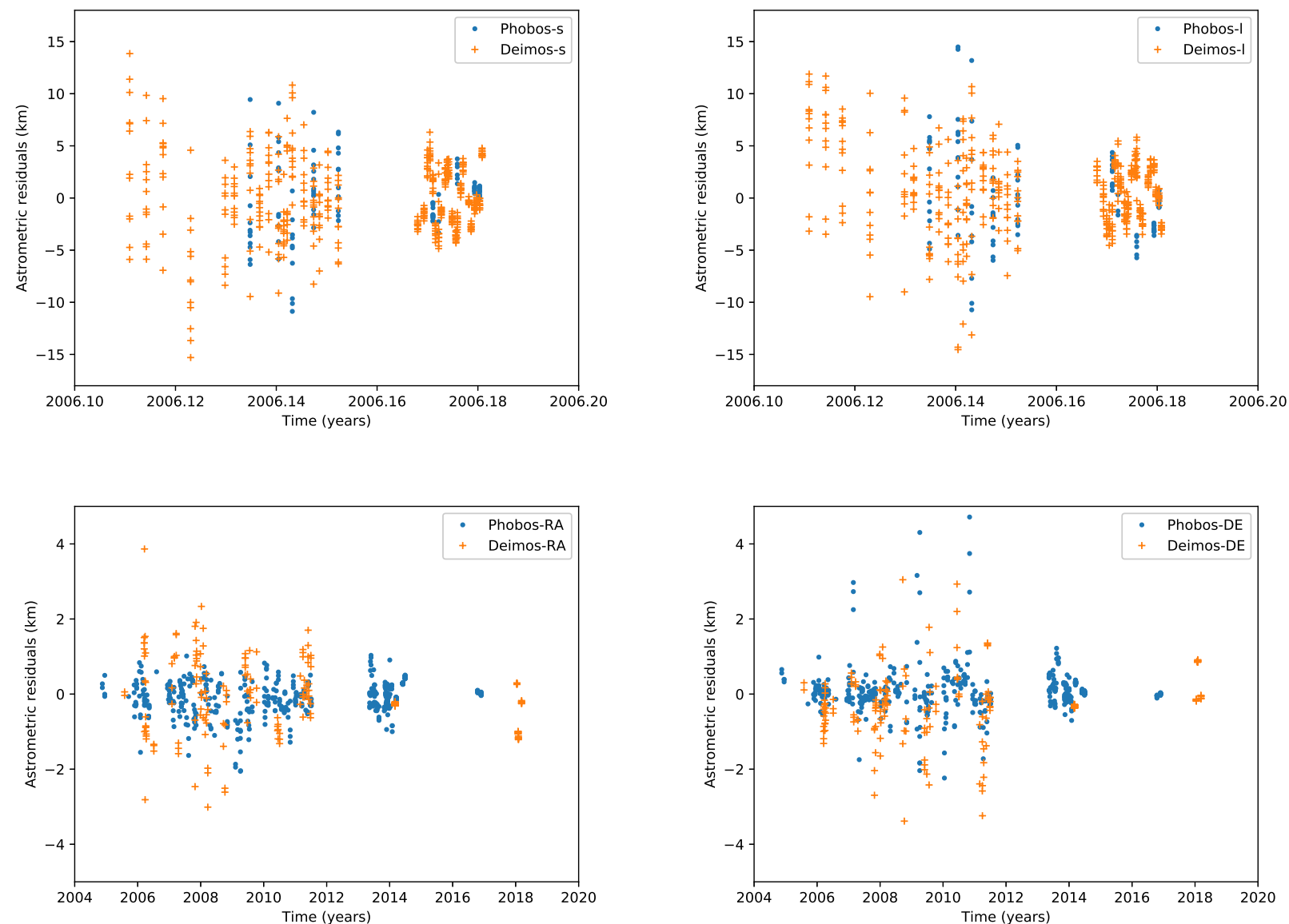

Fig. 3. Differences in distance after the fit between the model and (i) the MRO data (top), and (ii) the MEX data (bottom). The initial positions and velocities of the satellites, the Martian dissipation quality factor $Q$, and the forced libration amplitude of Phobos were fit here.

Table 3. Mean $(v)$ and standard deviation $(\sigma)$ on separation $s$ and position angle $p$ (multiplied by the separation) in seconds of degrees for each satellite.

\begin{tabular}{lrrrrrr}
\hline \hline Observations & $\begin{array}{r}v_{s} \\
\left({ }^{\prime \prime}\right)\end{array}$ & $\begin{array}{r}\sigma_{s} \\
\left({ }^{\prime \prime}\right)\end{array}$ & $\begin{array}{r}v_{p} \\
\left({ }^{\prime \prime}\right)\end{array}$ & $\begin{array}{c}\sigma_{p} \\
\left({ }^{\prime \prime}\right)\end{array}$ & $N$ & Satellite \\
\hline Morley (1989), Hall-Newcomb-Harkness & -0.0130 & 0.6717 & 0.0964 & 0.5627 & 112,110 & Phobos \\
US Naval Obs., Washington (before 1893) & 0.2381 & 0.6856 & 0.0141 & 0.5803 & 147,155 & Deimos \\
\hline Keeler (1888), Keeler (1890) & -0.1450 & 0.3827 & 0.2262 & 0.2840 & 59,103 & Phobos \\
Lick Observatory 1888, 1890 & 0.1310 & 0.6332 & 0.0200 & 0.5633 & 33,46 & Deimos \\
\hline Campbell (1892), Campbell (1895) & 0.0363 & 0.2243 & 0.0375 & 0.4388 & 380,111 & Phobos \\
Lick Observatory 1892, 1894 & 0.0083 & 0.3244 & -0.0739 & 0.4133 & 305,127 & Deimos \\
\hline Barnard (1897), Barnard (1910) & -0.0480 & 0.4101 & -0.2146 & 0.3935 & 63,50 & Phobos \\
Lick 1894, Yerkes 1909 & 0.3599 & 0.3210 & -0.0338 & 0.4927 & 18,12 & Deimos \\
\hline Morley (1989), Telescope:26-inch & -0.2101 & 0.3323 & -0.0299 & 0.3433 & 24,42 & Phobos \\
US Naval Obs., Washington (since 1893) & 0.0996 & 0.5336 & 0.0035 & 0.4300 & 52,53 & Deimos \\
\hline Newall (1895) & -0.1343 & 0.4268 & 0.0240 & 0.7057 & 30,3 & Phobos \\
Cambridge 1894 & 0.0000 & 0.0000 & 0.0000 & 0.0000 & 0,0 & Deimos \\
\hline Hall (1913) & 0.0426 & 0.4480 & 0.0387 & 0.2039 & 76,79 & Phobos \\
USNO 1909 & 0.0006 & 0.3957 & 0.0306 & 0.3637 & 85,85 & Deimos \\
\hline Jeffers (1925) & 0.0388 & 0.3907 & 0.0561 & 0.3054 & 41,32 & Phobos \\
Lick 1924 & 0.1918 & 0.3629 & -0.0064 & 0.2670 & 27,21 & Deimos \\
\hline Burton \& Hall (1923) & 0.0391 & 0.3168 & 0.1281 & 0.1972 & 37,38 & Phobos \\
USNO 1911-1922 & 0.0498 & 0.2226 & -0.0176 & 0.2165 & 50,50 & Deimos \\
\hline & & & & & &
\end{tabular}

Notes. $N$ is the number of observations by satellite (one number per coordinate). The year appearing next to each observatory name corresponds to the observed Mars opposition. 
Table 4. Mean $(v)$ and standard deviation $(\sigma)$ on right ascension and declination in seconds of degrees for each satellite.

\begin{tabular}{|c|c|c|c|c|c|c|}
\hline Observations & $\begin{array}{c}v_{\alpha \cos \delta} \\
\left({ }^{\prime \prime}\right)\end{array}$ & $\begin{array}{c}\sigma_{\alpha \cos \delta} \\
\left({ }^{\prime \prime}\right)\end{array}$ & $\begin{array}{r}v_{\delta} \\
\left({ }^{\prime \prime}\right)\end{array}$ & $\begin{array}{c}\sigma_{\delta} \\
\left({ }^{\prime \prime}\right)\end{array}$ & $N$ & Satellite \\
\hline Morley (1989), Hall-Newcomb-Harkness & -0.6395 & 0.0000 & 0.5384 & 0.0000 & 1,1 & Phobos \\
\hline US Naval Obs., Washington (before 1893) & -0.9176 & 0.4875 & 1.3264 & 0.1682 & 4,4 & Deimos \\
\hline Young (1880) & -1.0632 & 0.0000 & 0.0000 & 0.0000 & 1,0 & Phobos \\
\hline Princeton 1879 & 0.0000 & 0.0000 & 0.0000 & 0.0000 & 0,0 & Deimos \\
\hline Morley (1989), US Naval Obs., Washington (since 1893) & 0.0000 & 0.0000 & 0.0000 & 0.0000 & 0,0 & Phobos \\
\hline USNO 1894 & -1.0542 & 0.0000 & 0.3752 & 0.0000 & 1,1 & Deimos \\
\hline Newall (1895) & 0.0283 & 0.7718 & -0.2615 & 0.7440 & 23,22 & Phobos \\
\hline Cambridge 1894 & 0.0000 & 0.0000 & 0.0000 & 0.0000 & 0,0 & Deimos \\
\hline Kostinsky (1909), Kostinski (1913) & 0.0442 & 0.3596 & 0.0654 & 0.4658 & 23,24 & Phobos \\
\hline Pulkovo 1909 & 0.0029 & 0.5176 & -0.0445 & 0.4297 & 22,23 & Deimos \\
\hline Hall (1913) & -0.3370 & 0.3341 & 0.0571 & 0.3727 & 38,38 & Phobos \\
\hline USNO 1909 & -0.5577 & 0.4243 & 0.2008 & 0.2360 & 31,31 & Deimos \\
\hline Biesbroeck (1970) & -0.1590 & 0.1340 & -0.0217 & 0.0974 & 22,22 & Phobos \\
\hline Mc Donald 1956 & 0.1590 & 0.1340 & 0.0217 & 0.0974 & 22,22 & Deimos \\
\hline Robert et al. (2015), 61-inch & 0.0022 & 0.0532 & 0.0021 & 0.0514 & 216,216 & Phobos \\
\hline USNO (Flagstaff) 1967-1986 & 0.0018 & 0.0502 & -0.0009 & 0.0534 & 254,254 & Deimos \\
\hline Robert et al. (2015), 26-inch & 0.0002 & 0.0383 & 0.0024 & 0.0379 & 424,424 & Phobos \\
\hline USNO 1971-1997 & -0.0006 & 0.0345 & -0.0019 & 0.0343 & 450,450 & Deimos \\
\hline Kiseleva (1976) & -0.0135 & 0.2281 & -0.0280 & 0.2048 & 17,17 & Phobos \\
\hline Pulkovo 1973 & 0.0135 & 0.2281 & 0.0280 & 0.2048 & 17,17 & Deimos \\
\hline Kudryavtsev et al. (1992) & 0.0097 & 0.0750 & -0.0150 & 0.0901 & 660,660 & Phobos \\
\hline Shokin Majdanak 1988 & -0.0035 & 0.0753 & 0.0022 & 0.0718 & 639,639 & Deimos \\
\hline Bobylev et al. (1991), inter-satellite only & 0.0217 & 0.3642 & -0.0520 & 0.2276 & 50,50 & Phobos \\
\hline Pulkovo 1988 & 0.0297 & 0.1824 & -0.0171 & 0.2226 & 29,29 & Deimos \\
\hline Colas (1992) & -0.0215 & 0.0544 & -0.0012 & 0.0639 & 813,813 & Phobos \\
\hline Pic du Midi 1988 & 0.0215 & 0.0544 & 0.0012 & 0.0639 & 813,813 & Deimos \\
\hline Jones et al. (1989) & -0.0043 & 0.1191 & 0.0506 & 0.1150 & 154,154 & Phobos \\
\hline La Palma 1988 & -0.0574 & 0.1015 & -0.0293 & 0.0984 & 78,78 & Deimos \\
\hline Table Mountain Observatory (R.A. Jacobson, priv. comm.) & 0.0244 & 0.0443 & 0.0112 & 0.1070 & 6,6 & Phobos \\
\hline Table Mountain 2003 & -0.1105 & 0.1334 & -0.0355 & 0.0961 & 9,9 & Deimos \\
\hline Pascu (priv. comm.), B filter & 0.0116 & 0.0240 & 0.0104 & 0.0197 & 76,76 & Phobos \\
\hline USNO (Flagstaff) 2003 & -0.0116 & 0.0240 & -0.0104 & 0.0197 & 76,76 & Deimos \\
\hline Pascu (priv. comm.), V filter & 0.0025 & 0.0324 & 0.0105 & 0.0339 & 75,75 & Phobos \\
\hline USNO (Flagstaff) 2003 & -0.0025 & 0.0324 & -0.0105 & 0.0339 & 75,75 & Deimos \\
\hline $\mathrm{mm}$.), R filter & -0.0006 & 0.0267 & 0.0218 & 0.0363 & 56,56 & Phobos \\
\hline USNO (Flagstaff) 2003 & 0.0006 & 0.0267 & -0.0218 & 0.0363 & 56,56 & Deimos \\
\hline
\end{tabular}

Notes. $N$ is the number of observations by satellite.

Table 5. Mean $(v)$ and standard deviation $(\sigma)$ on sample and line in pixels and kilometers for each satellite.

\begin{tabular}{|c|c|c|c|c|c|c|c|c|c|c|}
\hline Observations & $\begin{array}{l}v_{\text {sample }} \\
\text { (pix) }\end{array}$ & $(\mathrm{km})$ & $\begin{array}{l}\sigma_{\text {sample }} \\
\text { (pix) }\end{array}$ & $(\mathrm{km})$ & $\begin{array}{c}v_{\text {line }} \\
\text { (pix) }\end{array}$ & $(\mathrm{km})$ & $\begin{array}{c}\sigma_{\text {line }} \\
\text { (pix) }\end{array}$ & $(\mathrm{km})$ & $N$ & Satellite \\
\hline \multirow[t]{2}{*}{ MRO (single) } & 0.0000 & 0.0000 & 0.0000 & 0.0000 & 0.0000 & 0.0000 & 0.0000 & 0.0000 & 0,0 & Phobos \\
\hline & 0.0031 & -0.0416 & 0.0722 & 3.6539 & 0.0081 & 0.8250 & 0.0614 & 3.500 & 376,376 & Deimos \\
\hline \multirow[t]{2}{*}{ MRO } & 0.0062 & -0.0607 & 0.0466 & 3.5902 & -0.0140 & 0.1301 & 0.0723 & 4.3938 & 103,103 & Phobos \\
\hline & -0.0062 & 0.0535 & 0.0466 & 3.5921 & 0.0140 & -0.1201 & 0.0723 & 4.4041 & 103,103 & Deimos \\
\hline
\end{tabular}

Notes. $N$ is the number of observations by a satellite. MRO (single) gathers data for which only one moon was observable at a time. 
V. Lainey et al:: Mars moon ephemerides after 14 years of Mars Express data

Table 6. Initial conditions and related uncertainties of Phobos and Deimos in the ICRF after fitting at initial epoch J2000 (Julian day 2451 545.0).

\begin{tabular}{lrrr}
\hline \hline Moon & & & \\
\hline Phobos & & & \\
$x, y, z$ & $-1989.71893421683 \pm 0.093$ & $-8743.02171778182 \pm 0.025$ & $-3181.65223492620 \pm 0.065$ \\
$v x, v y, v z$ & $1.84320501057858 \pm 3.8 \mathrm{E}-6$ & $-4.312869534231428 \mathrm{E}-2 \pm 2.6 \mathrm{E}-6$ & $-1.01836853901114 \pm 2.1 \mathrm{E}-6$ \\
$\begin{array}{l}\text { Deimos } \\
x, y, z\end{array}$ & $10366.3782389459 \pm 0.46$ & $-15747.7651557215 \pm 1.42$ & $-13945.1083381706 \pm 0.69$ \\
$v x, v y, v z$ & $1.04085057050574 \pm 1.09 \mathrm{E}-5$ & $0.843538311722695 \pm 1.09 \mathrm{E}-5$ & $-0.178915197575563 \pm 1.10 \mathrm{E}-5$ \\
\hline
\end{tabular}

Notes. Units are $\mathrm{km}$ and $\mathrm{km} \mathrm{sec}^{-1}$. All digits are kept for reproducibility of our results.

Table 7. Estimated forced libration in longitude of Phobos.

\begin{tabular}{lr}
\hline \hline Reference & $A(\mathrm{deg})$ \\
\hline Duxbury (1974) & 3. \\
Duxbury \& Callahan (1989) & $0.81 \pm 0.5$ \\
Borderies \& Yoder (1990) & 1.19 \\
Jacobson (2010) & $1.03 \pm 0.22$ \\
Willner et al. (2010) & $1.20 \pm 0.14$ \\
Willner et al. (2014) & 1.14 \\
Burmeister et al. (2018) & $1.14 \pm 0.03$ \\
Present work $(1 \sigma)$ & $1.09 \pm 0.01$
\end{tabular}

Notes. Duxbury (1974), Duxbury \& Callahan (1989), Willner et al. (2010), and Burmeister et al. (2018) used spacecraft imaging to solve directly for the physical libration of Phobos. Borderies \& Yoder (1990) and Willner et al. (2014) computed the libration of Phobos from the observed shape of Phobos assuming a homogeneous interior. Here, we follow Jacobson (2010) and determined the physical libration of Phobos from its effect on the Phobos orbit.

to add at least $k_{3}$. Nevertheless, it would be practically impossible to solve for two different $Q$, one associated with each tidal frequency.

The physical libration of Phobos was simultaneously found to be $A=1.09 \pm 0.01$ degrees (Table 7). This last value is at $5 \sigma$ below its theoretical value of 1.14 degrees (Willner et al. 2014) assuming homogeneity. Nevertheless, considering the very small error bar $(1 \%)$, a higher order degree should clearly be considered in the harmonics expansion of Phobos, and also an improved rotation model such as the one suggested by Rambaux et al. (2012), before any conclusion is drawn on the interior of Phobos.

\section{Precision versus accuracy}

While fitting our model to astrometric data, we discovered a disagreement between $\mathrm{MRO}^{2}$ and MEX data. In particular, while both sets did provide small residuals independently, they appeared to be strongly deteriorated when considered together for Deimos. Because both data sets roughly cover the same years, an error in the modeling sounds unlikely. Unfortunately, we were unable to use Phobos data to achieve a deeper understanding of the possible bias in these sets because the quality of the MRO data of Phobos is far lower. This bias was simultaneously observed at JPL and interpreted as a bias of 55."775 and $-7 . " 321$ in right ascension and declination in the MEX data, respectively (Robert Jacobson, priv. comm.). Because this bias 2 MRO astrometric data are available at: http://ssd.jpl.nasa.
gov/dat/sat/psf_mro.txt appeared to remain roughly constant over the years, MEX observations were supposed to be offset. However, because the MEX orbit is so specific, most MEX observations were performed in similar geometrical condition. As a consequence, a constant bias on the predicted position of Deimos may provide similar astrometric residuals.

A closer look at the MRO data showed that the errors of a few kilometers in Deimos observations were related to a difference only smaller than 0.2 pixel. A small difference like this is related to the scale of the image because Deimos was far away when it was observed for astrometric purposes by the MRO spacecraft. In particular, the Martian moons are known to have a complex shape, and the fit of their center of figure (assumed to be approximately equal to their center of mass) may be inaccurate when it is not properly resolved. Last but not least, using independent measurements, Ziese and Willner (2018) showed that our former solution NOE-4-2015-b (which did not use MRO data) agreed better for Deimos than mar097. We therefore again preferred the MEX data and removed the MRO data during the fitting procedure.

\section{Comparison of ephemerides and extrapolation precision}

We compared our ephemerides with our former solution NOE4-2015-b and with the ephemerides mar097 developed at JPL (Jacobson 2010). In particular, the NOE-4-2015-b solution used a dynamical model and observation sets similar to ours here. However, it did not benefit from the new MEX observations of Ziese and Willner (2018), nor from the unpublished observations we used here. Figure 4 shows differences for both moons in distance in 3D space. In the case of Phobos, the differences at the time close to 2010 are particularly small (at about a few kilometers) as a consequence of MEX data that have already been available in 2010. The differences then increase with time as a result of the small modeling and weight differences during the fitting procedure. The new MEX data used in this study provide confidence in the current ephemerides. On the other hand, in the case of Deimos, the differences increase linearly over time, as a consequence of the different treatment of the MRO data. This difference has previously been pointed out by Ziese and Willner (2018).

Figure 5 shows the formal uncertainty of our new ephemeris of Phobos and Deimos. The ephemeris uncertainty for Phobos is typically a few hundred meters for the present time. Because of its larger distance to MEX, the Deimos ephemeris is less well constrained, but it is expected to be precise within several hundred meters. The real accuracy of our new ephemerides will have to be confirmed by comparison with independent observational means. 

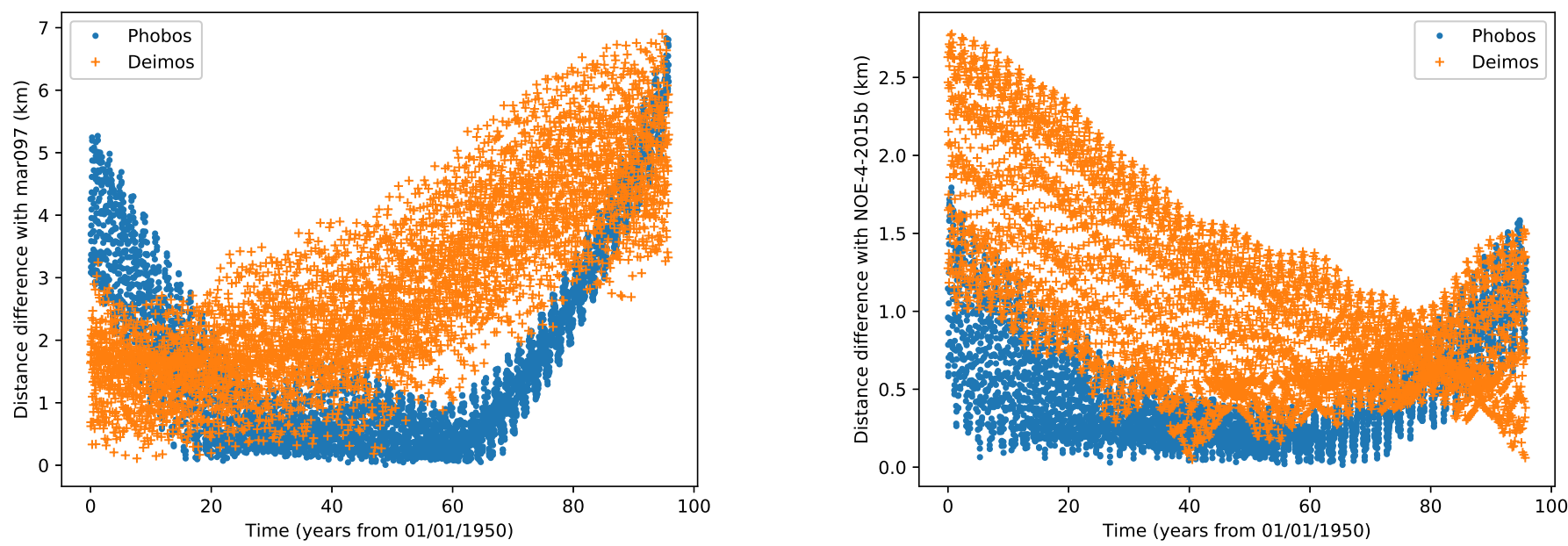

Fig. 4. Differences in distance in 3D space between our new ephemerides (NOE-4-2020) and (i) the JPL ephemerides mar097 (left), and (ii) our former ephemerides NOE-4-2015b (right).

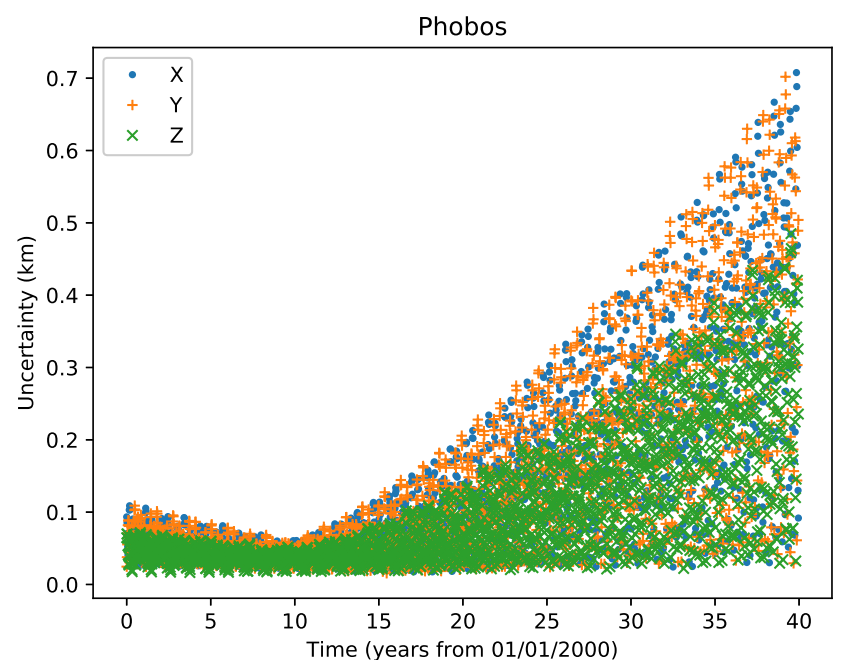

Fig. 5. $1 \sigma$ uncertainty of the ephemerides.

\section{Conclusions}

We developed new ephemerides of Phobos and Deimos, fitted to the longest observation time span, including recent unpublished astrometric data obtained by evaluating images taken by the HRSC onboard Mars Express. During the fitting process, we were able to solve for the physical libration of Phobos, which we found to be equal to $1.09 \pm 0.01$ degrees. Considering the very low uncertainty, this may suggest that higher order harmonics with an improved rotation model need to be considered in the future. We confirmed the inconsistency between recent Mars Reconnaissance Orbiter and Mars Express data for Deimos observations. Because observations with the Mars Reconnaissance Orbiter were performed from a large distance, the inconsistency is probably due to an erroneous determination of the center of figure. Our ephemerides NOE-4-2020 are available in SPICE format ${ }^{3}$.

Acknowledgements. The authors are indebted to O. Witasse, R. Jacobson and S. Le Maistre for fruitful discussions. This work has been supported by the European Community's Seventh Framework Program (FP7/2007-2013) under grant agreement 263466 for the FP7-ESPaCE project. The authors wish to thank

3 ftp://ftp.imcce.fr/pub/ephem/satel/NOE/MARS/2020/

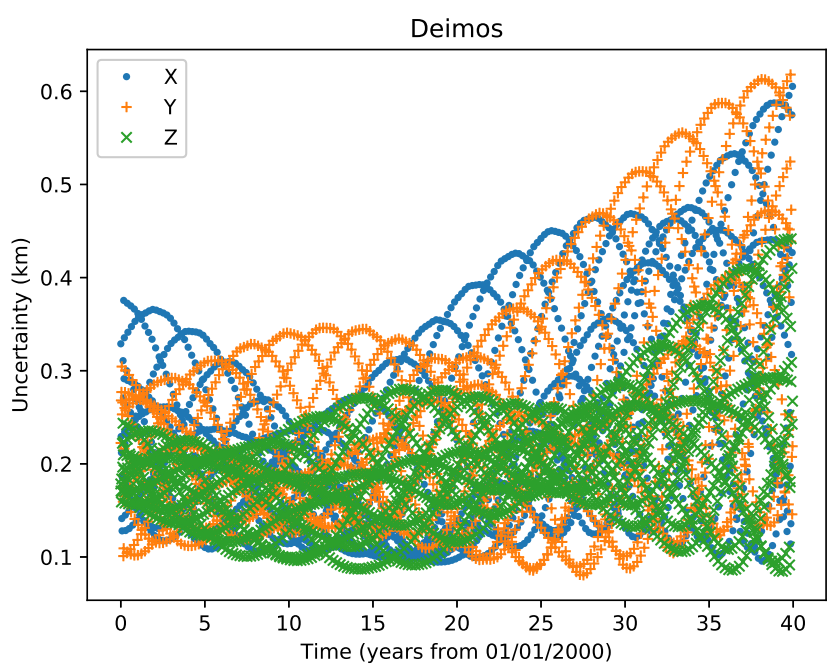

the HRSC Experiment team at DLR, Institute of Planetary Research, Berlin, and at Freie Universität Berlin, the HRSC Science Team, as well as the Mars Express Project teams at ESTEC, ESOC, and ESAC for their successful planning, acquisition, and release of image data to the community.

\section{References}

Archinal, B. A., Acton, C. H., A'Hearn, M. F., et al. 2018, Celest. Mech. Dyn. Astron., 130, 46

Archinal, B. A., Acton, C. H., Conrad, A., et al. 2019, Celest. Mech. Dyn. Astron., 131,61

Arlot, J.-E., \& Emelyanov, N. V. 2009, A\&A, 503, 631

Barnard, E. E. 1897, AJ, 17, 145

Barnard, E. E. 1910, AJ, 26, 69

Biesbroeck, G. V. 1970, Commun. Lunar Planet. Lab., 8, 179

Bobylev, V. V., Evdokimov, A. E., Kalinichenko, O. A, et al. 1991, Positional Photographic Observations of the Mars Satellites made at Pulkovo and Ordubad in 1988 (Izvestiia: Pulkovo, Glavnaia Astronomicheskaia Observatoriia) 207,37

Borderies, N., \& Yoder, C. F. 1990, A\&A, 233, 235

Burmeister, S., Willner, K., Schmidt, V., \& Oberst, J. 2018 J. Geodesy, 92, 963

Burton, H. E., \& Hall, Jr. A. 1923, AJ, 35, 113

Campbell, W. W. 1892, AJ, 12, 137

Campbell, W. W. 1895, AJ, 15, 1

Colas, F. 1992, A\&AS, 96, 485

de Cuyper, J.-P., de Decker, G., Winter, L., \& Zacharias, N. 2011, ASP Conf. Ser., 442, 301 
Duxbury, T. C. 1974 , Icarus, 23, 290

Duxbury, T. C., \& Callahan, J. D. 1988, A\&A, 201, 169

Duxbury, T. C., \& Callahan, J. D. 1989, A\&A, 216, 284

Duxbury, T., Hoffmann, H., Roatsch, T., et al. 2011, Mars Express Super Resolution Channel Image Restoration and Geometric Properties, Technical Note

Fienga, A., Avdellidou, C., \& Hanuš, J. 2020, MNRAS, 492, 589

Folkner, W. M., Williams, J. G., Boggs, D. H., Park, R. S., \& Kuchynka, P. 2014, Interplanet. Netw. Prog. Rep., 196, 1

Hall, Jr. A. 1913, AJ, 27, 163

Jacobson, R. A. 2010, AJ, 139, 668

Jaumann, R., Neukum, G., Behnke, T., et al. 2007, Planet. Space Sci., 55, 928

Jeffers, H. M. 1925, Lick Observ. Bull., 12, 4

Jones, D. H. P., Sinclair, A. T., \& Williams, I. P. 1989, MNRAS, 237, 15

Kaula, W. M. 1964, Rev. Geophys. Space Phys., 2, 661

Keeler, J. E. 1888, AJ, 8, 73

Keeler, J. E. 1890, AJ, 10, 89

Kiseleva, T. P. 1976, Izvestiya Glavnoj Astronomicheskoj Observatorii v Pulkove, 194, 127

Kiseleva, T. P., Panova, G. V., \& Kalinichenko, O. A. 1977, Izvestiya Glavnoj Astronomicheskoj Observatorii v Pulkove, 195, 49

Kolyuka, Y., Tikhonov, V., Ivanov, N., et al. 1991, A\&A, 244, 236

Konopliv, A. S., Park, R. S., \& Folkner, W. M. 2016, Icarus, 274, 253

Kostinsky, S. 1909, Astron. Nachr., 183, 7

Kostinski S. K. 1913, Mitteilungen der Nikolai-Hauptsternwarte zu Pulkowo, 5

Kuchynka, P., Folkner, W. M., Konopliv, A. S., et al. 2014, Icarus, 229, 340

Kudryavtsev, S. M., Shokin, Y. A., \& Yevstigneeva, N. 1992, from the observations during the 1988 opposition (in English) Preprint Sternberg State Astronomical Institute

Lainey, V., Dehant, V., \& Pätzold, M. 2007, A\&A, 465, 1075

Lainey, V., Jacobson, R. A., Tajeddine, R., et al. 2017, Icarus, 281, 286

Lainey, V., Noyelles, B., Cooper, N., et al. 2019, Icarus, 326, 48
Michael, G., \& Neukum, G. 2009, Lunar Planet. Sci. Conf., 40, 1851

Morley, T. A. 1989, A\&AS, 77, 209

Newall, H. F. 1895, MNRAS, 55, 348

Oberst, J., Schwarz, G., Behnke, T., et al. 2008, Planet. Space Sci., 56, 473

Pascu, D. 1977, IAU Colloq., 28, 63

Pascu, D. 1978, Vistas Astron., 22, 141

Pascu, D. 1979, in Natural and Artificial Satellite Motion, eds. P. E. Nacozy, \& S. Ferraz-Mello (Austin: University of Texas Press), 17

Pascu, D. 2012, in Proceedings of NAROO-GAIA Workshop: A new reduction of old observations in the Gaia era

Pascu, D., Erard, S., Thuillot, W., \& Lainey, V. 2014, Planet. Space Sci., 102, 2

Pasewaldt, A., Oberst, J., Willner, K., et al. 2012, A\&A, 545, A144

Pasewaldt, A., Oberst, J., Willner, K., et al. 2015, A\&A, 580, A28

Rambaux, N., Castillo-Rogez, J. C., Le Maistre, S., \& Rosenblatt, P. 2012, A\&A, 548, A 14

Robert, V., de Cuyper, J.-P., Arlot, J.-E., et al. 2011, MNRAS, 415, 701

Robert, V., Lainey, V., Pascu, D., et al. 2014, A\&A, 572, A104

Robert, V., Lainey, V., Pascu, D., et al. 2015, A\&A, 582, A36

Rosenblatt, P., Lainey, V., Maistre, S. Le, et al. 2008, Planet. Space Sci. 56, 1043 Sharpless, B. P. 1945, AJ, 51, 185

Stark, A. Willner, K., Burmeister, S., \& Oberst, J. 2017, Eur. Planet. Sci. Congress, (EPSC2017-868)

Thomas, P. C., Yoder, C. F., Synnott, S. P., et al. 2000, NASA Planetary Data System, EAR-A-5-DDR-SHAPE-MODELS-V2.1

Thuillot, W., Lainey, V., Dehant, V., et al. 2012, ASP Conf. Ser., 461, 659

Willner, K., Oberst, J., Wählisch, M., et al. 2008, A\&A, 488, 361

Willner, K., Oberst, J., Hussmann, H., et al. 2010, Earth Planet. Sci. Lett., 294, 541

Willner, K., Shi, X., \& Oberst, J. 2014, Planet. Space Sci., 102, 51

Yang, X., Yan, J. G., Andert, T., et al. MNRAS, 490, 2007

Young, C. A. 1880 The Observatory, 3, 270

Ziese, R., \& Willner, K. 2018 A\&A, 614, A15 